\title{
无溶剂条件下 $\mathrm{LiNTf}_{2}$ 催化的醛和酮的非手性硅氰化反应研究
}

\author{
王宏社* 曾君娥 \\ (宝鸡文理学院化学化工系 陕西省植物化学重点实验室 宝鸡 721013)
}

\begin{abstract}
摘要 以 $\mathrm{LiNT}_{2}$ 为催化剂, 在无溶剂条件下, 醛或酮与三甲基氰硅烷(TMSCN)室温发生加成反应, 得到相应的 $\alpha$-氰基 硅醚. 该法操作简便、反应条件温和、收率高、催化剂易回收和可以重复使用.
\end{abstract}

关键词 $\mathrm{LiNTf}_{2}$; 醛; 酮; 硅氨化反应; 无溶剂

\section{Solvent-Free Achiral Cyanosilylation of Aldehydes and Ketones Catalyzed by $\mathrm{LiNTf}_{2}$}

\author{
Wang, Hongshe* Zeng, June \\ (Shaanxi Key Laboratory for Phytochemistry, Department of Chemistry and Chemical Engineering, \\ Baoji University of Arts and Sciences, Baoji 721013)
}

\begin{abstract}
An efficient method for the addition of trimethylsilyl cyanide (TMSCN) to various aldehydes and ketones has been described using $\operatorname{LiNTf}_{2}(1 \mathrm{~mol} \%)$ as a catalyst at room temperature under solvent-free conditions. The advantages of this method are easy work-up, mild reaction conditions, high yields and the catalyst exhibited remarkable reusable activity.

Keywords LiNTf 2 ; aldehyde; ketone; cyanosilylation; solvent-free
\end{abstract}

在有机合成中, 氰醇特别是旋光活性的氧醇是一类 重要的中间体, 通过它可以衍生出许多在医药、农药及 其它精细有机化学上有用的化合物. 通常, 氧醇可以通 过三甲基氰硅烷(TMSCN)与醛或酮的亲核加成反应再 经过水解得到 ${ }^{[1,2]}$. TMSCN 作为一种非常有用的亲核氧 化试剂, 已经被广泛应用于有机合成中 ${ }^{[3]}$. 它具有亲核 性强、对映选择性良好、稳定、易于使用与保存和对反 应条件要求温和等优点. TMSCN 与醛或酮进行亲核加 成得到的 $\alpha$-氧基硅醚, 还可以用来合成 $\alpha, \beta$-不饱和腈和 $\beta$-氨基醇等, 而且可用于保护羰基 ${ }^{[4]}$. 近年来, 已有很 多催化剂被用于催化醛或酮的非手性硅氰化反应, 例如 $\mathrm{Cu}\left(\mathrm{ClO}_{4}\right)_{2} \cdot 6 \mathrm{H}_{2} \mathrm{O}^{[5]}, \mathrm{HClO}_{4} / \mathrm{SiO}_{2}{ }^{[6]}$, 氮杂环卡宾 ${ }^{[7]}, \mathrm{LiCl} /$ $\mathrm{THF}^{[8]}, \mathrm{Au}^{[9]}, \mathrm{SbCl}_{3}{ }^{[10]},\left[\left(\mathrm{Ph}_{3} \mathrm{P}\right)_{2} \mathrm{~N}\right]^{+} \mathrm{Cl}^{-[11]}, \mathrm{Sc}(\mathrm{OTf})_{3}{ }^{[12]}$, $\mathrm{NbF}_{5}{ }^{[13]}, \mathrm{MgO}^{[14]}, \mathrm{SO}_{4}{ }^{2-} / \mathrm{ZrO}_{2}{ }^{[15]}, \mathrm{ZnI}_{2}{ }^{[16]}$ 和 $\mathrm{LiClO}_{4}{ }^{[17]}{ }^{\text {等. }}$ 虽然以上催化剂都取得了一定的效果, 但是有的催化剂 催化的硅氰化反应存在反应时间长、催化剂用量多和使 用有机溶剂等缺点. 同时, 很多手性催化剂被用于催化 醛或酮的对映选择性硅氧化反应 ${ }^{[18 ~ 24]}$.
双(三氟甲基磺酰)亚胺金属盐 $\left[\mathrm{M}\left(\mathrm{NTf}_{2}\right)_{n}\right]$ 作为一类 高效的 Lewis 酸催化剂已被广泛应用于有机合成 ${ }^{[25 ~ 30], ~}$ 并显示出了优良的催化性能. $\mathrm{M}\left(\mathrm{NTf}_{2}\right)_{n}$ 在有机反应中的 催化性能，一方面是因为分子中阴、阳离子的弱配位作 用, 另一方面是因为全氟烷基磺酰亚胺负离子同时是非 常好的亲电和亲核离去基团. 正是由于 $\mathrm{M}\left(\mathrm{NTf}_{2}\right)_{n}$ 分子 结构上的特点, 才使它们展现出高的 Lewis 酸酸性. 与 传统的 Lewis 酸催化剂相比, $\mathrm{M}\left(\mathrm{NTf}_{2}\right)_{n}$ 具有环境友好、 耐水性好、可回收循环使用等特点 ${ }^{[25]}$. 为拓展 $\mathrm{M}\left(\mathrm{NTf}_{2}\right)_{n}$ 在有机合成上的应用范围, 在前期工作基础上 ${ }^{[31,32]}$, 本 文以商业上容易得到且价格较低的 $\mathrm{LiNTf}_{2}$ 为催化剂, 研究了无溶剂条件下醛和酮的非手性硅氰化反应. 结果 表明, 在室温下反应就可以顺利进行, 并且催化剂用量 少(1 mol\%) (Eq. 1).

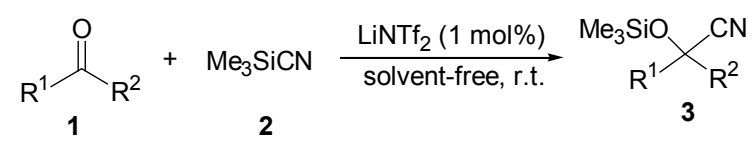

*E-mail: hongshewang@126.com

Received October 24, 2011; revised December 20,2011; published online January 11, 2012

Project supported by the Scientific Research Program of Shaanxi Provincial Education Deparment (No. 09JK332) and the Key Project of Baoji University of Arts and Sciences (No. ZK1053).

陕西省教育厅科研计划(No. 09JK332)和宝鸡文理学院重点(No. ZK1053)资助项目. 


\section{1 结果与讨论}

以苯甲醛和 TMSCN 的硅氰化反应为例, 首先考察 了商业上容易得到的四种双(三氟甲基磺酰)亚胺金属盐 $\operatorname{Mg}\left(\mathrm{NTf}_{2}\right)_{2}, \operatorname{LiNTf}_{2}, \mathrm{Yb}\left(\mathrm{NTf}_{2}\right)_{3}$ 和 $\operatorname{Sc}\left(\mathrm{NTf}_{2}\right)_{3}$ 的催化活性 (表 1). 从表 1 可以看出, 在这四种双(三氟甲基磺酰)亚 胺金属盐中, $\mathrm{LiNTf}_{2}$ 的催化活性最高, 反应 $10 \mathrm{~min}$, 收 率可以达到 $98 \%$. 另外, 在四种催化剂中, $\mathrm{LiNTf}_{2}$ 价格 最低, 没有毒性 ${ }^{[33]}$, 而 $\mathrm{Yb}\left(\mathrm{NTf}_{2}\right)_{3}$ 和 $\mathrm{Sc}\left(\mathrm{NTf}_{2}\right)_{3}$ 价格高, 所以, $\mathrm{LiNTf}_{2}$ 是催化苯甲醛与 $\mathrm{TMSCN}$ 反应的首选催化 剂. 同时, 我们比较了 $\mathrm{LiNTf}_{2}$ 与文献报道的其它锂盐的 催化效果. 当以 $\mathrm{LiClO}_{4}$ 为催化剂时, 虽然苯甲醛和 TMSCN 的硅氰化反应的产率可以达 $100 \%$, 但是反应时 间较长, 且催化剂用量是 $100 \mathrm{~mol} \%$; 当以 $\mathrm{LiCl}$ 为催化 剂时，虽然用量较少(3 $\mathrm{mol} \%$ ), 但是反应时间也较长, 同时, 催化剂需要使用 $\mathrm{LiCl}$ 的 THF 溶液, 且其溶液还要 用声波处理, 操作比较麻烦.

表 1 不同催化剂催化苯甲醛和 TMSCN 的硅氰化反应 ${ }^{a}$

Table 1 Cyanosilylation of benzaldehyde with TMSCN catalyzed by different catalysts

\begin{tabular}{clcc}
\hline Entry & \multicolumn{1}{c}{ Catalyst $/ \mathrm{mol} \%$} & Time $/$ min & Yield $^{c} \%$ \\
\hline 1 & $\mathrm{LiNTf}_{2} / 1$ & 10 & 98 \\
2 & $\mathrm{Mg}\left(\mathrm{NTf}_{2}\right)_{2} / 1$ & 20 & 95 \\
3 & $\mathrm{Yb}\left(\mathrm{NTf}_{2}\right)_{3} / 3$ & 45 & 74 \\
4 & $\mathrm{Sc}\left(\mathrm{NTf}_{2}\right)_{3} / 3$ & 45 & 60 \\
5 & $\mathrm{LiClO}_{4}{ }^{117]} / 100$ & 30 & 100 \\
$6^{b}$ & $\mathrm{LiCl}^{b} \mathrm{THF}^{[8]} / 3$ & 60 & 94 \\
\hline
\end{tabular}

${ }^{a}$ The reaction was performed with $1 \mathrm{mmol}$ of benzaldehyde and $1.2 \mathrm{mmol}$ of TMSCN at room temperature under solvent-free conditions. ${ }^{b} \mathrm{LiCl}$ was used as a $29.5 \mathrm{mmol} / \mathrm{L}$ solution of THF. ${ }^{c}$ Isolated yield.

其次, 考察了不同溶剂 $\left(\mathrm{CH}_{2} \mathrm{Cl}_{2}, \mathrm{CH}_{3} \mathrm{OH}, \mathrm{THF}\right.$, DMF，甲苯)以及无溶剂条件对反应的影响(表 2). 由表 2 可以看出, 当以 $1 \mathrm{~mol} \%$ 的 $\mathrm{LiNTf}_{2}$ 为催化剂, 在室温下, 以无溶剂条件时产率最高. 当反应在有机溶剂中进行 时, 产品收率较低, 其中, 以 $\mathrm{CH}_{3} \mathrm{OH}$ 为溶剂时, 产率只 有 $16 \%$, 这可能是因为溶剂使反应物和催化剂的浓度降 低. 因此, 无论从反应效果, 还是从环境角度来看, 无 溶剂条件是 $\mathrm{LiNTf}_{2}$ 催化硅氰化反应的首选反应条件.

最后, 考察了 $\mathrm{LiNTf}_{2}$ 用量对反应的影响(表 3). 没 有催化剂存在时, 即使反应时间达 $360 \mathrm{~min}$, 反应也几 乎不发生. 在同样反应条件下，当加入 $0.1 \mathrm{~mol} \%$ 的 LiNTf $_{2}$ 时, 反应速度加快, 产率有了较大提高. 增加催 化剂的用量, 反应产率会提高. 当催化剂用量为 $1 \mathrm{~mol} \%$ 时, 产率达到 98\%, 进一步增加催化剂用量收率并没有 提高, 因此, 采用 $1 \mathrm{~mol} \%$ 的 $\mathrm{LiNTf}_{2}$ 作为最佳催化剂用 量.

另外, 我们还考察了催化剂的重复使用情况. 每次
表 2 不同溶剂对苯甲醛和 TMSCN 反应的影响 ${ }^{a}$

Table 2 Effect of different solvents on the reaction of benzaldehyde with TMSCN

\begin{tabular}{clcc}
\hline Entry & Solvent & Time/min & Yield $^{c} / \%$ \\
\hline 1 & $\mathrm{CH}_{2} \mathrm{Cl}_{2}$ & 20 & 95 \\
2 & $\mathrm{CH}_{3} \mathrm{OH}$ & 90 & 16 \\
3 & $\mathrm{THF}$ & 20 & 92 \\
4 & $\mathrm{DMF}$ & 40 & 87 \\
5 & Toluene & 70 & 71 \\
$6^{b}$ & None & 10 & 98 \\
\hline
\end{tabular}

${ }^{a}$ The reaction was carried out in different solvents at room temperature with 1 mol\% LiNTf $_{2}{ }^{b}$ The reaction was carried out at room temperature under solvent-free conditions with $1 \mathrm{~mol} \% \operatorname{LiNTf}_{2} .{ }^{c}$ Isolated yield.

表 3 催化剂用量对苯甲醛和和 TMSCN 反应的影响 ${ }^{a}$

Table 3 Effect of different amount of $\operatorname{LiNTf}_{2}$ on the reaction of benzaldehyde with TMSCN

\begin{tabular}{cccc}
\hline Entry & LiNTf $_{2} / \mathrm{mol}^{2}$ & Time $/$ min & Yield $^{c} \%$ \\
\hline 1 & 0.1 & 90 & 52 \\
2 & 0.5 & 25 & 90 \\
3 & 1.0 & 10 & 98 \\
4 & 3.0 & 5 & 98 \\
5 & 5.0 & 5 & 98 \\
$6^{b}$ & 0 & 360 & $<1$ \\
\hline
\end{tabular}

${ }^{a}$ The reaction was carried out at room temperature under solvent-free conditions. ${ }^{b}$ Reaction without catalyst. ${ }^{c}$ Isolated yield.

实验完毕, 将回收的催化剂 $\mathrm{LiNTf}_{2}$ 在 $120{ }^{\circ} \mathrm{C}$ 干燥 $3 \mathrm{~h}$ 后, 冷却，再重复使用. 仍以苯甲醛与 TMSCN 在室温下的 反应为例，研究了 $\mathrm{LiNTf}_{2}$ 重复使用时的催化活性. 实验 结果表明(表 4), $\mathrm{LiNTf}_{2}$ 重复使用 5 次，其催化活性仍然 较高, 只是反应时间有所增长.

表 $4 \mathrm{LiNTf}_{2}$ 在苯甲醛与 TMSCN 的反应中的重复使用 Table 4 The reuse of $\operatorname{LiNTf}_{2}$ for the reaction of benzaldehyde with TMSCN

\begin{tabular}{ccc}
\hline Cycle & Time/min & Yield $^{a} / \%$ \\
\hline 0 & 10 & 98 \\
1 & 10 & 98 \\
2 & 10 & 96 \\
3 & 10 & 95 \\
4 & 15 & 93 \\
5 & 15 & 90 \\
\hline
\end{tabular}

${ }^{a}$ Isolated yield.

确定了较佳的反应条件后, 以 $1 \mathrm{~mol} \%$ 的 $\operatorname{LiNTf}_{2}$ 为 催化剂, 在无溶剂条件下, 研究了 TMSCN 与不同醛和 酮在室温下进行的硅氧化反应(表 5). 由表 5 可以看出, 所有醛和酮均可与 TMSCN 顺利发生反应，收率都在 $92 \%$ 以上. $\mathrm{LiNTf}_{2}$ 对芳香醛、脂肪醛、杂环醛和 $\alpha, \beta$-不饱 和醛都有很好的催化活性. 对于芳香醛来说, 苯环上的 取代基并没有对反应产生明显影响(表 5, Entries 1 5). 呋喃甲醛和 3-吡啶甲醛的产率分别是 94\%和 99\%(表 5, Entries 6,7)， $\alpha, \beta$-不饱和醛的产率为 99\%(表 5, Entry 8). 
脂肪醛也容易发生反应，且产率高(表 5, Entries 9, 10). 与醛相比, 酮进行反应所需时间虽然较长, 但是也有极 好的产率(表 5, Entries 11 15).

表 $5 \mathrm{LiNTf}_{2}$ 催化的醛和酮的硅氭化反应 ${ }^{a}$

Table 5 Cyanosilylation of aldehydes and ketones with TMSCN catalyzed by $\mathrm{LiNTf}_{2}$

\begin{tabular}{clcccc}
\hline Entry & \multicolumn{1}{c}{$\mathrm{R}^{1}$} & $\mathrm{R}^{2}$ & Product & Time/min & Yield $^{b} \%$ \\
\hline 1 & $\mathrm{Ph}$ & $\mathrm{H}$ & $\mathbf{3 a}$ & 10 & 98 \\
2 & $2-\mathrm{CH}_{3} \mathrm{C}_{6} \mathrm{H}_{4}$ & $\mathrm{H}$ & $\mathbf{3 b}$ & 10 & 96 \\
3 & $4-\mathrm{CH}_{3} \mathrm{OC}_{6} \mathrm{H}_{4}$ & $\mathrm{H}$ & $\mathbf{3 c}$ & 10 & 92 \\
4 & $2-\mathrm{ClC}_{6} \mathrm{H}_{4}$ & $\mathrm{H}$ & $\mathbf{3 d}$ & 10 & 96 \\
5 & $4-\mathrm{NO}_{2} \mathrm{C}_{6} \mathrm{H}_{4}$ & $\mathrm{H}$ & $\mathbf{3 e}$ & 10 & 98 \\
6 & $2-\mathrm{Furyl}_{7}$ & $\mathrm{H}$ & $\mathbf{3 f}$ & 10 & 94 \\
7 & $3-\mathrm{Pyridyl}$ & $\mathrm{H}$ & $\mathbf{3 g}$ & 10 & 99 \\
8 & $(E)-\mathrm{PhCH}_{2} \mathrm{CH}$ & $\mathrm{H}$ & $\mathbf{3 h}$ & 15 & 99 \\
9 & $\mathrm{CH}_{3}\left(\mathrm{CH}_{2}\right)_{5}$ & $\mathrm{H}$ & $\mathbf{3 i}$ & 10 & 94 \\
10 & $\mathrm{Cyclohexyl}_{11}$ & $\mathrm{H}$ & $\mathbf{3 j}$ & 10 & 98 \\
12 & $\mathrm{Ph}_{12}$ & $\mathrm{CH}$ & $\mathbf{3 k}$ & 30 & 95 \\
13 & $4-\mathrm{ClC}_{6} \mathrm{H}_{4}$ & $\mathrm{Ph}_{3}$ & $\mathbf{3 l}$ & 45 & 92 \\
14 & $4-\mathrm{CH}_{3} \mathrm{C}_{6} \mathrm{H}_{4}$ & $\mathbf{3 m}$ & 30 & 96 \\
15 & $\mathrm{CH}_{3}\left(\mathrm{CH}_{2}\right)_{2}$ & $\mathrm{CH}_{3}$ & $\mathbf{3 n}$ & 30 & 95 \\
\hline
\end{tabular}

${ }^{a}$ All the reactions were carried out with $1 \mathrm{~mol} \% \mathrm{LiNTf}_{2}$ at room temperature under solvent-free conditions. ${ }^{b}$ Isolated yield.

参照文献[7, 8, 13], $\operatorname{LiNTf}_{2}$ 催化醛或酮的硅氭化反 应的可能反应机理如 Scheme 1 所示. 首先催化剂 $\mathrm{LiTf}_{2} \mathrm{~N}$ 与 $\mathrm{TMSCN}$ 发生加成反应生成高价硅化合物 $\mathbf{4}$, 然后 4 与醛或酮反应生成中间体 $\mathbf{5}$, 最后 5 分解生成 $\operatorname{LiNTf}_{2}$ 和目标化合物 3.

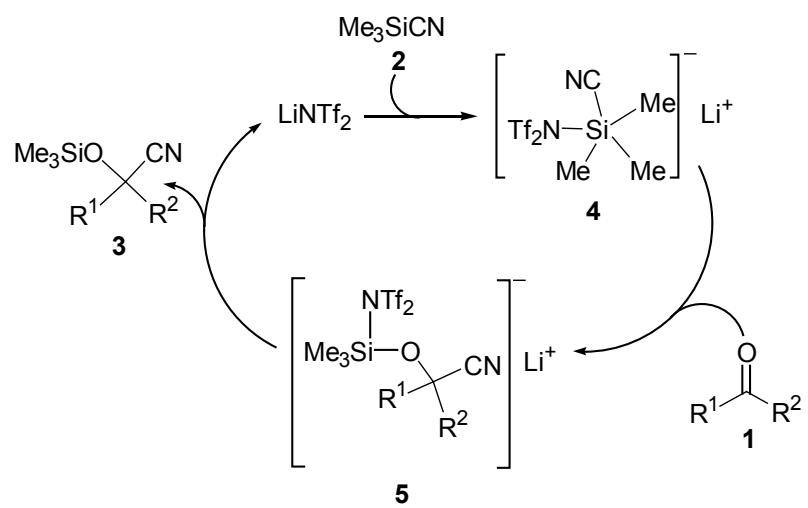

Scheme 1

\section{2 实验部分}

\section{1 仪器与试剂}

Nicolet FTIR-750 型红外光谱仪; Bruker 400 核磁共 振仪 (TMS 为内标, $\mathrm{CDCl}_{3}$ 为溶剂); 高分辨质谱仪 $\mathrm{Q}-\mathrm{Tof}$ micro. 所有试剂均为市售化学纯或分析纯.

\section{2 实验方法}

在圆底烧瓶中加入醛或酮 $(1,1 \mathrm{mmol}), \mathrm{TMSCN}(2$, $1.2 \mathrm{mmol}$ )和 $\operatorname{LiNTf}_{2}(0.01 \mathrm{mmol}, 1 \mathrm{~mol} \%)$, 在室温摚拌反 应, TLC 检测反应终点. 反应结束后, 用石油醚 $(5 \mathrm{~mL} \times$ 3)萃取, 有机相用无水 $\mathrm{Na}_{2} \mathrm{SO}_{4}$ 干燥, 减压蒸除溶剂得粗 产物. 粗产物通过柱层析 $[V$ (已烷 $): V($ 乙酸乙酯 $)=9$ : 1 ]得纯品 3.

2-(三甲基硅氧基)-2-苯基乙腈 (3a $)^{[8]}$ : 无色油状液 体. ${ }^{1} \mathrm{H}$ NMR $\left(400 \mathrm{MHz}, \mathrm{CDCl}_{3}\right) \delta: 0.27\left(\mathrm{~s}, 9 \mathrm{H}, 3 \mathrm{CH}_{3}\right)$, $5.81(\mathrm{~s}, 1 \mathrm{H}, \mathrm{CHCN}), 7.36 \sim 7.48(\mathrm{~m}, 5 \mathrm{H}, \mathrm{ArH}) ;{ }^{13} \mathrm{C} \mathrm{NMR}$ $\left(100 \mathrm{MHz}, \mathrm{CDCl}_{3}\right) \delta:-0.2,63.3,119.3,126.5,129.2$, 129.6, 136.2; IR (neat) $v: 3081,2261(\mathrm{CN}), 753 \mathrm{~cm}^{-1}$. HRMS calcd for $\mathrm{C}_{11} \mathrm{H}_{15} \mathrm{NOSi} 205.0923$, found 205.0911.

2-(三甲基硅氧基)-2-(2-甲基苯基)乙腈(3b)：无色油 状液体, b.p. $77 \sim 79{ }^{\circ} \mathrm{C} / 0.13 \mathrm{kPa} ;{ }^{1} \mathrm{H}$ NMR $(400 \mathrm{MHz}$, $\left.\mathrm{CDCl}_{3}\right) \delta: 0.23\left(\mathrm{~s}, 9 \mathrm{H}, 3 \mathrm{CH}_{3}\right), 2.42\left(\mathrm{~s}, 3 \mathrm{H}, 3 \mathrm{CH}_{3}\right), 5.51(\mathrm{~s}$, $1 \mathrm{H}, \mathrm{CHCN}), 7.20 \sim 7.29(\mathrm{~m}, 3 \mathrm{H}, \mathrm{ArH}), 7.51 \sim 7.56(\mathrm{~m}$, $1 \mathrm{H}, \mathrm{ArH}) ;{ }^{13} \mathrm{C}$ NMR $\left(100 \mathrm{MHz}, \mathrm{CDCl}_{3}\right) \delta:-0.3,18.5$, 61.6, 118.9, 126.3, 127.2, 129.4, 131.2, 134.0, 135.8; IR (neat) $v: 3076,2242(\mathrm{CN}), 756 \mathrm{~cm}^{-1}$. HRMS calcd for $\mathrm{C}_{12} \mathrm{H}_{17} \mathrm{NOSi} 219.1080$, found 219.1068.

2-(三甲基硅氧基)-2-(4-甲氧基苯基)乙腈 $(\mathbf{3 c})^{[8]}$ : 无 色油状液体. ${ }^{1} \mathrm{H}$ NMR $\left(400 \mathrm{MHz}, \mathrm{CDCl}_{3}\right) \delta: 0.23(\mathrm{~s}, 9 \mathrm{H}$, $\left.3 \mathrm{CH}_{3}\right), 3.61\left(\mathrm{~s}, 3 \mathrm{H}, \mathrm{CH}_{3}\right), 5.32(\mathrm{~s}, 1 \mathrm{H}, \mathrm{CHCN}), 6.70(\mathrm{~d}$, $J=6.8 \mathrm{~Hz}, 2 \mathrm{H}, \mathrm{ArH}), 7.16(\mathrm{~d}, J=6.8 \mathrm{~Hz}, 2 \mathrm{H}, \mathrm{ArH}) ;{ }^{13} \mathrm{C}$ NMR $\left(100 \mathrm{MHz}, \mathrm{CDCl}_{3}\right) \delta:-0.2,55.5,63.9,114.6$, 119.8, 128.0, 128.5, 160.4; IR (neat) v: 3050, 2241 (CN), $750 \mathrm{~cm}^{-1}$. HRMS calcd for $\mathrm{C}_{12} \mathrm{H}_{17} \mathrm{NO}_{2} \mathrm{Si} 235.1029$, found 235.1018 .

2-(三甲基硅氧基)-2-(2-氯苯基)乙腈 $(\mathbf{3 d})^{[8]}$ : 无色油 状液体. ${ }^{1} \mathrm{H}$ NMR $\left(400 \mathrm{MHz}, \mathrm{CDCl}_{3}\right) \delta: 0.25(\mathrm{~s}, 9 \mathrm{H}$, $\left.3 \mathrm{CH}_{3}\right), 5.56(\mathrm{~s}, 1 \mathrm{H}, \mathrm{CHCN}), 7.19 \sim 7.28(\mathrm{~m}, 3 \mathrm{H}, \mathrm{ArH})$, $7.62 \sim 7.65(\mathrm{~m}, 1 \mathrm{H}, \mathrm{ArH}) ;{ }^{13} \mathrm{C}$ NMR $\left(100 \mathrm{MHz}, \mathrm{CDCl}_{3}\right) \delta$ : $-0.3,61.2,118.4,127.6,128.8,130.3,131.5,132.4$, 134.2; IR (neat) $v: 3105,2244(\mathrm{CN}), 749 \mathrm{~cm}^{-1}$. HRMS calcd for $\mathrm{C}_{11} \mathrm{H}_{14} \mathrm{ClNOSi} 239.0533$, found 239.0546.

2-(三甲基硅氧基)-2-(4-硝基苯基)乙腈(3e $)^{[15]}$ : 浅黄 色油状液体. ${ }^{1} \mathrm{H}$ NMR $\left(400 \mathrm{MHz}, \mathrm{CDCl}_{3}\right) \delta: 0.27$ (s, 9H, $\left.3 \mathrm{CH}_{3}\right), 5.53(\mathrm{~s}, 1 \mathrm{H}, \mathrm{CHCN}), 7.60 \sim 7.64(\mathrm{~d}, J=8.6 \mathrm{~Hz}, 2 \mathrm{H}$, ArH ), 8.20 (d, $J=8.6 \mathrm{~Hz}, 2 \mathrm{H}, \mathrm{ArH}) ;{ }^{13} \mathrm{C}$ NMR $(100 \mathrm{MHz}$, $\left.\mathrm{CDCl}_{3}\right) \delta:-0.3,62.6,118.4,124.2,127.5,143.1,148.5$; IR (neat) $v: 3108,2352(\mathrm{CN}), 746 \mathrm{~cm}^{-1}$. HRMS calcd for $\mathrm{C}_{11} \mathrm{H}_{14} \mathrm{~N}_{2} \mathrm{O}_{3} \mathrm{Si} 250.0899$, found 250.0891 .

2-(三甲基硅氧基)-2-(呋喃基)乙腈(3f) ${ }^{[13]}$ : 无色油状 液体. ${ }^{1} \mathrm{H}$ NMR $\left(400 \mathrm{MHz}, \mathrm{CDCl}_{3}\right) \delta: 0.19\left(\mathrm{~s}, 9 \mathrm{H}, 3 \mathrm{CH}_{3}\right)$, 
$5.44(\mathrm{~s}, 1 \mathrm{H}, \mathrm{CHCN}), 6.16 \sim 6.20(\mathrm{~m}, 1 \mathrm{H}, \operatorname{ArH}), 6.32(\mathrm{~d}$, $J=3.6 \mathrm{~Hz}, 1 \mathrm{H}, \mathrm{ArH}), 7.25 \sim 7.28(\mathrm{~m}, 1 \mathrm{H}, \mathrm{ArH}) ;{ }^{13} \mathrm{C} \mathrm{NMR}$ $\left(100 \mathrm{MHz}, \mathrm{CDCl}_{3}\right) \delta:-0.4,57.8,110.3,111.1,117.3$, 144.2, 148.7; IR (neat) $v: 3086,2353(\mathrm{CN}), 757 \mathrm{~cm}^{-1}$. HRMS calcd for $\mathrm{C}_{9} \mathrm{H}_{13} \mathrm{NO}_{2} \mathrm{Si}$ 195.0716, found 195.0723.

2-(三甲基硅氧基)-2-(3-吡啶基)乙腈(3g $)^{[17]}$ : 浅黄色 油状液体. ${ }^{1} \mathrm{H}$ NMR $\left(400 \mathrm{MHz}, \mathrm{CDCl}_{3}\right) \delta: 0.25(\mathrm{~s}, 9 \mathrm{H}$, $\left.3 \mathrm{CH}_{3}\right), 5.50$ (s, $\left.1 \mathrm{H}, \mathrm{CHCN}\right), 7.35$ (dd, $J=4.8,7.8 \mathrm{~Hz}, 1 \mathrm{H}$, ArH), 7.86 (d, $J=7.8 \mathrm{~Hz}, 1 \mathrm{H}, \mathrm{ArH}), 8.60$ (d, $J=4.8 \mathrm{~Hz}$, $1 \mathrm{H}, \mathrm{ArH}), 8.65$ (s, $1 \mathrm{H}, \mathrm{ArH}) ;{ }^{13} \mathrm{C} \mathrm{NMR}\left(100 \mathrm{MHz}, \mathrm{CDCl}_{3}\right)$ $\delta: 1.9,60.5,118.6,124.3,132.7,134.8,147.2,149.9$; IR (neat) $v$ : $3080,2346(\mathrm{CN}), 751 \mathrm{~cm}^{-1}$. HRMS calcd for $\mathrm{C}_{10} \mathrm{H}_{14} \mathrm{~N}_{2} \mathrm{OSi}$ 206.0875, found 206.0867.

(E)-2-(三甲基硅氧基)-4-苯基-3-丁烯腈(3h $)^{[8]}$ : 无色 油状液体. ${ }^{1} \mathrm{H}$ NMR $\left(400 \mathrm{MHz}, \mathrm{CDCl}_{3}\right) \delta: 0.26(\mathrm{~s}, 9 \mathrm{H}$, $3 \mathrm{CH}_{3}$ ), 5.22 (d, $\left.J=6.0 \mathrm{~Hz}, 1 \mathrm{H}, \mathrm{CHCN}\right), 6.16$ (dd, $J=6.0$, $15.9 \mathrm{~Hz}, 1 \mathrm{H}, \mathrm{CHCH}=\mathrm{CH}), 6.83(\mathrm{~d}, J=15.9 \mathrm{~Hz}, 1 \mathrm{H}$, $\mathrm{CH}=\mathrm{CHPh}), 7.28 \sim 7.36(\mathrm{~m}, 5 \mathrm{H}, \mathrm{ArH}) ;{ }^{13} \mathrm{C}$ NMR $(100$ $\left.\mathrm{MHz}, \mathrm{CDCl}_{3}\right) \delta:-0.2,62.5,118.5,123.2,126.8,128.7$, 128.8, 133.7, 134.9; IR (neat) $v$ : 3090, $2254(\mathrm{CN}), 753$ $\mathrm{cm}^{-1}$. HRMS calcd for $\mathrm{C}_{13} \mathrm{H}_{17} \mathrm{NOSi} 231.1079$, found 231.1085 .

2-(三甲基硅氧基)辛腈 (3i) ${ }^{[6]}$ : 无色油状液体. ${ }^{1} \mathrm{H}$ $\operatorname{NMR}\left(400 \mathrm{MHz}, \mathrm{CDCl}_{3}\right) \delta: 0.22\left(\mathrm{~s}, 9 \mathrm{H}, 3 \mathrm{CH}_{3}\right), 0.81(\mathrm{t}$, $\left.J=6.8 \mathrm{~Hz}, 3 \mathrm{H}, \mathrm{CH}_{3}\right), 1.21 \sim 1.30\left(\mathrm{~m}, 6 \mathrm{H}, 3 \mathrm{CH}_{2}\right), 1.35 \sim$ $1.40\left(\mathrm{~m}, 2 \mathrm{H}, \mathrm{CH}_{2}\right), 1.67 \sim 1.72\left(\mathrm{~m}, 2 \mathrm{H}, \mathrm{CH}_{2}\right), 4.28(\mathrm{t}, J=$ $6.5 \mathrm{~Hz}, 1 \mathrm{H}, \mathrm{CHCN}) ;{ }^{13} \mathrm{C} \mathrm{NMR}\left(100 \mathrm{MHz}, \mathrm{CDCl}_{3}\right) \delta:-$ $0.4,14.1,22.6,24.3,28.8,31.5,36.3,52.4,120.2$; IR (neat) $v$ : $2358(\mathrm{CN}), 742 \mathrm{~cm}^{-1}$. HRMS calcd for $\mathrm{C}_{11} \mathrm{H}_{23} \mathrm{~N}-$ OSi 213.1549, found 213.1562.

2-三甲基硅氧基-2-环已基乙腈 $(\mathbf{3 j})^{[8]}$ : 无色油状液 体. ${ }^{1} \mathrm{H}$ NMR $\left(400 \mathrm{MHz}, \mathrm{CDCl}_{3}\right) \delta: 0.22\left(\mathrm{~s}, 9 \mathrm{H}, 3 \mathrm{CH}_{3}\right)$, $1.05 \sim 1.27\left(\mathrm{~m}, 6 \mathrm{H}, 3 \mathrm{CH}_{2}\right), 1.60 \sim 1.79\left(\mathrm{~m}, 4 \mathrm{H}, 2 \mathrm{CH}_{2}\right)$, $2.00 \sim 2.03(\mathrm{~m}, 1 \mathrm{H}, \mathrm{CH}), 4.18(\mathrm{~d}, J=6.6 \mathrm{~Hz}, 1 \mathrm{H}, \mathrm{CHCN})$; ${ }^{13} \mathrm{C}$ NMR $\left(100 \mathrm{MHz}, \mathrm{CDCl}_{3}\right) \delta:-0.5,25.5,26.1,28.1$, 28.3, 42.9, 66.7, 119.6; IR (neat) $v: 2350(\mathrm{CN}), 745 \mathrm{~cm}^{-1}$. HRMS calcd for $\mathrm{C}_{11} \mathrm{H}_{21} \mathrm{NOSi} 211.1392$, found 211.1384.

2-(三甲基硅氧基)-2-苯基丙腈 $(\mathbf{3 k})^{[11]}$ : 无色油状液 体. ${ }^{1} \mathrm{H}$ NMR (400 MHz, $\left.\mathrm{CDCl}_{3}\right) \delta: 0.18\left(\mathrm{~s}, 9 \mathrm{H}, 3 \mathrm{CH}_{3}\right)$, 1.89 (s, 3H, $\left.\mathrm{CH}_{3}\right), 7.30 \sim 1.35(\mathrm{~m}, 3 \mathrm{H}, \mathrm{ArH}), 7.48 \sim 7.51$ (m, 2H, ArH); ${ }^{13} \mathrm{C}$ NMR $\left(100 \mathrm{MHz}, \mathrm{CDCl}_{3}\right) \delta: 1.1,33.7$, $71.5,121.4,124.6,128.5,128.6,141.7$; IR (neat) $v: 3088$, $2363(\mathrm{CN}), 755 \mathrm{~cm}^{-1}$. HRMS calcd for $\mathrm{C}_{12} \mathrm{H}_{17} \mathrm{NOSi}$ 219.1079, found 219.1072.

2-(三甲基硅氧基)-2-二苯基乙腈(3I) ${ }^{[11]}$ : 无色油状
液体. ${ }^{1} \mathrm{H}$ NMR $\left(400 \mathrm{MHz}, \mathrm{CDCl}_{3}\right) \delta: 0.13\left(\mathrm{~s}, 9 \mathrm{H}, 3 \mathrm{CH}_{3}\right)$, $7.31 \sim 1.37$ (m, 6H, ArH), 7.44 7.48 (m, $4 \mathrm{H}, \mathrm{ArH}) ;{ }^{13} \mathrm{C}$ $\operatorname{NMR}\left(100 \mathrm{MHz}, \mathrm{CDCl}_{3}\right) \delta: 0.9,76.5,120.4,125.7,128.6$, 128.7, 141.9; IR (neat) $v: 3101,2370(\mathrm{CN}), 761 \mathrm{~cm}^{-1}$. HRMS calcd for $\mathrm{C}_{17} \mathrm{H}_{19} \mathrm{NOSi} 281.1236$, found 281.1245.

2-(三甲基硅氧基)-2-(4-氯苯基)丙腈 $(\mathbf{3 m})^{[14]}$ : 无色 油状液体. ${ }^{1} \mathrm{H}$ NMR $\left(400 \mathrm{MHz}, \mathrm{CDCl}_{3}\right) \delta: 0.18(\mathrm{~s}, 9 \mathrm{H}$, $\left.3 \mathrm{CH}_{3}\right), 1.85$ (s, 3H, $\left.\mathrm{CH}_{3}\right), 7.35$ (d, $\left.J=8.6 \mathrm{~Hz}, 2 \mathrm{H}, \mathrm{ArH}\right)$, $7.49(\mathrm{~d}, J=8.6 \mathrm{~Hz}, 2 \mathrm{H}, \mathrm{ArH}) ;{ }^{13} \mathrm{C}$ NMR $(100 \mathrm{MHz}$, $\left.\mathrm{CDCl}_{3}\right) \delta: 1.1,33.6,71.2,121.2,126.2,128.9,134.7$, 140.7; IR (neat) $v: 3093,2365(\mathrm{CN}), 759 \mathrm{~cm}^{-1}$. HRMS calcd for $\mathrm{C}_{12} \mathrm{H}_{16} \mathrm{ClNOSi} 253.0690$, found 253.0677.

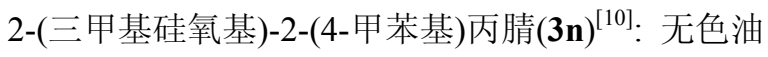
状液体. ${ }^{1} \mathrm{H}$ NMR $\left(400 \mathrm{MHz}, \mathrm{CDCl}_{3}\right) \delta: 0.16(\mathrm{~s}, 9 \mathrm{H}$, $\left.3 \mathrm{CH}_{3}\right), 1.82$ (s, $\left.3 \mathrm{H}, \mathrm{CH}_{3}\right), 2.36$ (s, $\left.3 \mathrm{H}, \mathrm{CH}_{3}\right), 7.17 \sim 7.19$ (m, 2H, ArH), $7.40 \sim 7.42(\mathrm{~m}, 2 \mathrm{H}, \mathrm{ArH}) ;{ }^{13} \mathrm{C}$ NMR $(100$ $\left.\mathrm{MHz}, \mathrm{CDCl}_{3}\right) \delta: 1.1,21.1,33.5,71.6,121.5,124.6,129.4$, 138.7, 139.1; IR (neat) $v: 3084,2360(\mathrm{CN}), 753 \mathrm{~cm}^{-1}$. HRMS calcd for $\mathrm{C}_{13} \mathrm{H}_{19} \mathrm{NOSi} 233.1236$, found 233.1248.

2-甲基-2-(三甲基硅氧基)戊腈(3o): 无色油状液体. b.p. $93 \sim 95{ }^{\circ} \mathrm{C} / 0.93 \mathrm{kPa} ;{ }^{1} \mathrm{H}$ NMR $\left(400 \mathrm{MHz}, \mathrm{CDCl}_{3}\right) \delta$ : $0.22\left(\mathrm{~s}, 9 \mathrm{H}, 3 \mathrm{CH}_{3}\right), 0.93\left(\mathrm{t}, J=7.2 \mathrm{~Hz}, 3 \mathrm{H}, \mathrm{CH}_{3}\right), 2.36$ (s, $\left.3 \mathrm{H}, \mathrm{CH}_{3}\right), 1.35 \sim 1.61\left(\mathrm{~m}, 5 \mathrm{H}, \mathrm{CH}_{2} \mathrm{CH}_{3}\right), 1.65 \sim 1.72(\mathrm{~m}$, $\left.2 \mathrm{H}, \mathrm{CH}_{2}\right) ;{ }^{13} \mathrm{C}$ NMR $\left(100 \mathrm{MHz}, \mathrm{CDCl}_{3}\right) \delta: 1.3,13.8,17.7$, 28.9, 45.6, 69.7, 122.3; IR (neat) $v: 2351(\mathrm{CN}), 747 \mathrm{~cm}^{-1}$. HRMS calcd for $\mathrm{C}_{9} \mathrm{H}_{19} \mathrm{NOSi} 185.0916$, found 185.0905 .

\section{References}

[1] Breuer, M.; Ditrich, K.; Habicher, T.; Hauer, B.; Kebeler, M.; Sturmer, R.; Zelinski, T. Angew. Chem., Int. Ed. 2004, 43, 788.

[2] Ryu, D. H.; Corey, E. J. J. Am. Chem. Soc. 2004, 126, 8106.

[3] Luo, H. Chin. J. Org. Chem. 2011, 31, 1 (in Chinese). (罗海南, 有机化学, 2011, 31, 1.)

[4] Gregory, R. H. J. Chem. Rev. 1999, 99, 3649.

[5] Majhi, A.; Kim, S. S.; Kim, H. S. Appl. Organomet. Chem. 2008, $22,407$.

[6] Heydari, A.; Mamani, L. Appl. Organomet. Chem. 2008, 22, 12.

[7] Song, J. J.; Gallou, F.; Reeves, J. T.; Tan, Z.; Yee, N. K.; Senanayake, C. H. J. Org. Chem. 2006, 71, 1273.

[8] Kurono, N.; Yamaguchi, M.; Suzuki, K.; Ohkuma, T. J. Org. Chem. 2005, 70, 6530.

[9] Cho, W. K.; Lee, J. K.; Kang, S. M.; Chi, Y. S.; Lee, H.-S.; Choi, I. S. Chem. Eur. J. 2007, 13, 6351.

[10] Pourmousavi, S. A.; Salahshornia, H. Bull. Korean Chem. Soc. 2011, 32, 1575.

[11] Lacour, M.-A.; Rahier, N. J.; Taillefer, M. Chem. Eur. J. 2011, 17, 12276.

[12] Park, B. Y.; Ryu, K. Y.; Park, J. H.; Lee, S. Green Chem. 2009, 11, 946.

[13] Kim, S. S.; Rajagopal, G. Synthesis 2007, 215.

[14] kantam, M. L.; Mahendar, K.; Sreedhar, B.; Kumar, K. V.; Choudary, B. M. Synth. Commun. 2008, 38, 3919. 
[15] Thirupathi, B.; Patil, M. K.; Reddy, B. M. Appl. Catal. A: Gen. 2010, 384, 147.

[16] Wang, X.-L.; Wang, Y.-X.; Bian, Z.-X. Acta Chim. Sinica 2007, 65, 1670 (in Chinese) (王晓丽, 王艳学, 边占喜, 化学学报, 2007, 65, 1670.)

[17] Azizi, N.; Saidi, M. R. J. Organomet. Chem. 2003, 688, 283.

[18] Geng, X.-H.; Zhou, H.; Chen, P.-R.; Xu, Q. Chin. J. Org. Chem. 2008, 28, 1157 (in Chinese). (耿晓红, 周辉, 陈沛然, 徐青, 有机化学, 2008, 28, 1157.)

[19] Wang, W.; Liu, X.; Lin, L.; Feng, X. Eur. J. Org. Chem. 2010, 4751 .

[20] Zhang, Z.; Wang, Z.; Zhang, R.; Ding, K. Angew. Chem., Int. Ed. 2010, 49, 6746 .

[21] Kurono, N.; Arai, K.; Uemura, M.; Ohkuma, T. Angew. Chem., Int. Ed. 2008, 47, 6643.

[22] Ryu, D. H.; Corey, E. J. J. Am. Chem. Soc. 2005, 127, 5384.

[23] Liu, X.; Qin, B.; Zhou, X.; He, B.; Feng, X. J. Am. Chem. Soc. 2005, 127, 12224.

[24] Dang, D.; Wu, P.; He, C.; Xie, Z.; Duan, C. J. Am. Chem. Soc. 2010, 132, 14321

[25] Yuan, Y.-B.; Nie, J. Chin. J. Org. Chem. 2004, 24, 857 (in Chi- nese).

(袁余斌, 聂进, 有机化学, 2004, 24, 857.)

[26] Wang, L.-Q.; Nie, J.; Li, X.-Y.; Zhang, Z.-B.; Yin, F. Chin. J. Org. Chem. 2004, 24, 778 (in Chinese).

(王丽琼，聂进，李小永，张正波，尹飞，有机化学，2004，24， 778.)

[27] Asao, N.; Aikawa, H. J. Org. Chem. 2006, 71, 5249.

[28] Sweis, R. F.; Schramm, M. P.; Kozmin, S. A. J. Am. Chem. Soc. 2004, 126, 7442 .

[29] Earle, M. J.; Hakala, U.; McAuley, B. J.; Nieuwenhuyzen, M.; Ramani, A.; Seddon, K. R. Chem. Commun. 2004, 1368.

[30] Grau, F.; Heumann, A.; Dunach, E. Angew. Chem., Int. Ed. 2006, 45,7285 .

[31] Wang, H.-S.; Zhao, L.-F. Chin. J. Org. Chem. 2005, 25, 869 (in Chinese).

(王宏社, 赵立芳, 有机化学, 2005, 25, 869.)

[32] Wang, H.; Zeng, J. Chin. J. Org. Chem. 2010, 30, 1072 (in Chinese).

(王宏社, 曾君娥, 有机化学, 2010, 30, 1072.)

[33] Cossy, J.; Bellosta, V.; Hamoir, C.; Desmurs, J.-R. Tetrahedron Lett. 2002, 43, 7083. 